Revue d'histoire de l'enfance " irrégulière »

Le Temps de l'histoire

19 | 2017

Abandon d'enfants et parents abandonneurs, $X I X X^{e}-\left.X X\right|^{e}$ siècles

\title{
La difficile prise en charge de l'enfance abandonnée dans la Biscaye du début du XIX siècle
}

Difficulties in caring for foundlings in early 19th-century Biscay province

Sylvie Hanicot-Bourdier

\section{(2) OpenEdition \\ Journals}

Édition électronique

URL : https://journals.openedition.org/rhei/4014

DOI : $10.4000 /$ rhei.4014

ISSN : $1777-540 \mathrm{X}$

Éditeur

Presses universitaires de Rennes

\section{Édition imprimée}

Date de publication : 22 novembre 2017

Pagination : 25-46

ISBN : 978-2-7535-6467-1

ISSN : $1287-2437$

Référence électronique

Sylvie Hanicot-Bourdier, « La difficile prise en charge de l'enfance abandonnée dans la Biscaye du début du xıx siècle », Revue d'histoire de l'enfance « irrégulière » [En ligne], 19 | 2017, mis en ligne le 27 novembre 2019, consulté le 08 septembre 2021. URL : http://journals.openedition.org/rhei/4014 DOI : https://doi.org/10.4000/rhei.4014 


\section{La difficile prise en charge de l'enfance abandonnée dans la Biscaye du début du $x^{e} x^{e}$ siècle}

La création en 1798 et 1806 de deux maisons d'accueil donne à voir l'impact des lois et des règlements édictés par les autorités royales et provinciales dans la prise en charge des enfants abandonnés d'une région qui conserve une autonomie administrative et financière jusqu'à la fin du XIX $x^{\mathrm{e}}$ siècle. Elle témoigne d'une amélioration des conditions d'existence de l'enfance délaissée biscayenne. Mais, faute de moyens financiers, le sort des orphelins biscayens est, au début du $\mathrm{XIX}^{\mathrm{e}}$ siècle, encore extrêmement précaire. L'ouverture de structures réservées à la seule enfance illégitime concourt à évincer les enfants nés hors mariage de leur foyer d'origine. Elle génère une augmentation des délaissements et paradoxalement la surmortalité de nourrissons que les autorités provinciales sont censées protéger. Elle oblige de surcroît les parents légitimes, qui ne souhaitent pas ou ne peuvent pas conserver leur enfant, à recourir à l'exposition. Aussi, qu'ils soient naturels ou légitimes, l'abandon des plus petits devient, dans la Biscaye du XIX siècle, un fait de société indiscutable.

The establishment of two children's homes in Biscay province in 1798 and 1806 demonstrates the impact of the regulations issued by the royal and provincial authorities for the care of abandoned children. It is evidence of an improvement in the living conditions of abandoned children in a region that retained some administrative and financial autonomy until the end of the 19th century. As a result of a lack of funds, the fate of these orphans was still highly precarious at the start of the 19th century. The opening of homes for illegitimate children only had the effect of removing children born out of wedlock from their original family homes. It caused an increase in abandonment and, paradoxically, higher mortality among infants the provincial authorities were supposed to be protecting. It also forced legitimate parents who would not or could not keep their children to leave them to be found. The abandonment of the newly born, whether legitimate or illegitimate, became an undeniable social phenomenon in 19th-century Biscay.

Mots-clés: enfance abandonnée, prise en charge, Biscaye, Espagne, XIX siècle. Keywords: abandoned children, foundling care, Biscay, Spain, 19th century.

\author{
Sylvie \\ Hanicot-Bourdier \\ Professeure des \\ universités en civili- \\ sation de l'Espagne \\ à l'université de \\ Lorraine. Membre du \\ centre de recherches \\ "Littératures \\ Imaginaire Sociétés » \\ (LIS - EA 7305).
}


1. Sablayrolles Élisabeth, L'enfance abandonnée à Strasbourg au XVIII siècle et la fondation de la Maison des enfants-trouvés, Strasbourg, Librairie Istra, 1976, p. 76.

2. Montalvo Tomás, Práctica política y economía de Expósitos, en que se describen su origen, $y$ calidades, resolviéndose las dudas, que pueden ofrecerse en esta materia, y juntamente se declara el gobierno doméstico, que en sus Hospitales se debe observar, Granada, Imprenta de la Santísima Trinidad, 1700.

3. Bilbao Antonio, Destrucción y conservación de los expósitos. Idea de la perfección de este ramo de policía. Modo breve de poblar la España, p. 12.

4. Ibid., p. 66-107.

5. García Santiago, Breve instrucción sobre el modo de conservar los niños expósitos aprobada por el Real Tribunal de Protomedicato, Madrid, M. González, 1794.

6. Murcia Pedro Joachin, Discurso político sobre la importancia y necesidad de los Hospicios, Casas de expósitos y Hospitales, que tienen todos los Estados, y particularmente España, Madrid, Imprenta la Viuda de Ibarra, 1798.

7. ÚRIz Joaquín Xavier Causas prácticas de la muerte de los niños expósitos en sus primeros años, remedio en su origen

de un tan grave mal, $y$ modo de formarles útiles a la religión y al estado, con notable aumento de la población, fuerzas y riquezas de España, Pamplona, Imprenta de Josef de Rada, 1801.

8. Murcia Pedro Joachin, Discurso político..., op. cit. p. 66-67.
A u xiII ${ }^{\mathrm{e}}$ siècle, l'effroyable mortalité des enfants abandonnés suscite l'indignation des esprits éclairés français qui, particulièrement sensibles aux problèmes de l'éducation et de l'enfance marginalisée, dénoncent ce massacre d'innocents. Invoquant des raisons humanitaires, religieuses et économiques, les hommes des Lumières exigent une intervention de l'État au profit d'une meilleure prise en charge de l'enfance délaissée ${ }^{1}$.

En Espagne, dès 1700, Fray Tomás de Montalvo consacre 731 pages à l'insoutenable situation des enfants abandonnés du royaume ${ }^{2}$. Il faut toutefois attendre la fin du XvIII ${ }^{e}$ siècle pour que cette question devienne l'une des principales préoccupations des intellectuels espagnols. Le plus connu d'entre eux, Antonio de Bilbao, dénonce en 1789 le décès de milliers d'enfants que la monarchie espagnole maintient dans un état de délaissement et de souffrance intolérable 3 . Considérant que l'insuffisance des moyens et le manque criant d'établissements d'accueil sont à l'origine de cette insupportable hécatombe, Antonio de Bilbao exige des pouvoirs publics un effort financier et l'ouverture de nouveaux centres d'accueil ${ }^{4}$. En 1794, Santiago García revient sur la nécessité de construire de nouveaux orphelinats ${ }^{5}$. Pedro Joachin de Murcia en $1798^{6}$, puis Joaquín Xavier de Úriz en $1801^{7}$ reprennent également le combat d'Antonio de Bilbao. Le premier s'insurge contre le décès de milliers de nouveau-nés qui, faute de soins adéquats, périssent chaque année aux quatre coins du royaume $e^{8}$. Deux années plus tard, Joaquín Xavier de Úriz, evêque de Pampelune, évoque des milliers de nourrissons 
qui, « enfant [s] de Dieu? " et " portraits vivants du Rédempteur ${ }^{10}$ ", périssent par manque de soins, ce qui offense la religion chrétienne et l'humanité, et entrave la prospérité du royaume ${ }^{11}$. Signe que les mentalités tardent à changer, en 1805, Alberto de Megino revient sur l'état de délabrement des orphelinats espagnols ${ }^{12}$ et évalue à 21000 le nombre de nouveau-nés qui décèdent chaque année dans ces maisons de torture ${ }^{13}$.

C'est dans ce nouveau contexte de prise de conscience que sont fondés, à la fin du XVIII ${ }^{\mathrm{e}}$ siècle et au début du siècle suivant, les premiers orphelinats de Biscaye, une province espagnole située sur la frange littorale du nord de la péninsule Ibérique et cernée par le Guipúzcoa à l'est, la Cantabrie à l'ouest et l'Alava au sud.

En Espagne, de nombreuses études livrent un riche panorama de la situation de l'enfance délaissée du XVI ${ }^{\mathrm{e}}$ au XIX ${ }^{\mathrm{e}}$ siècles ${ }^{14}$. Mais l'histoire des enfants abandonnés de Biscaye reste à faire. Combler ce vide historiographique à une époque charnière, celle du passage de la société d'Ancien Régime au premier libéralisme espagnol du début du XIX ${ }^{\mathrm{e}}$ siècle, donne à voir l'impact des lois et des règlements édictés par les autorités royales et provinciales dans la prise en charge des enfants abandonnés d'une région qui conserve une autonomie administrative et financière jusqu'à la fin du XIX ${ }^{e}$ siècle ${ }^{15}$.

Pour mener à bien cette étude, nous disposons d'un fonds archivistique totalement inexploité, le fonds "expósitos de Bizkaia ", entreposé dans l'Archivo Foral de Bizkaia. D'un accès excessivement limité, cette documentation ne peut être consultée qu'avec une autorisation spéciale ${ }^{16}$. Ces restrictions d'accès ne sont pas spécifiques à cette
9. ÚRIZ Joaquín Xavier, Causas prácticas..., op. cit. p. 3

10. Ibid., p. 5

11. Ibid., p. 6

12. MEGINo Alberto, La demauxesia aumentación del pueblo por los medios de procurar que no mueran 50000 personas que según un cálculo prudencial, y bien formado, se pierden anualmente en las Casas de Espósitos, en los Ospicios, y en las Cárceles de España, Venice, Antonio Curti, 1805, p. 2 .

13. Ibid., p. 3.

14. Parmi les plus connues, mentionnons les recherches de León Carlos Álvarez Santaló sur les orphelins sévillans, celles de Claude Larquié sur Madrid et celles d'Hélène Tropé sur Valence. Álvarez SAntaló León Carlos, Marginación social y mentalidad en Andalucía Occidental: Expósitos en Sevilla (16131910), Sevilla, Consejería de Cultura, 1980; LARQuí́ Claude, "Le poids financier de l'abandon de l'enfant à Madrid (1650-1700)", Actes du colloque international de Rome, Publications de l'École française de Rome, 1991, volume 140, no 1, p. 785-813; Tropé Hélène, La formation des enfants orphelins à
Valence ( $x v^{e}-x v I^{e}$ siècles). Le cas du Collège impérial Saint-Vincent-Ferrier, Paris Presses de la Sorbonne Nouvelle, 1998.

\section{Malgré l'effort de} centralisation entrepris par l'État espagnol tout au long des $x \|_{I I}^{e}$ et $x I^{e}$ siècles, les trois provinces basques et la Navarre conservent un régime foral jusqu'en 1876 . Le maintien de ce système accorde à la Biscaye une entière liberté en matière de répartition et de recouvrement de l'impôt.

16. Son existence nous a été révélée par hasard. Après avoir parcouru l'ensemble des fonds municipaux et provinciaux des Archives forales de Biscaye à

la recherche de toute information ayant un lien avec l'enfance abandonnée, nous nous sommes étonnée de l'absence de certains documents. Nous nous sommes demandée pourquoi ces fonds ne conservaient aucune trace des orphelins recueillis aux $X V I I{ }^{e}$ et $x I x^{e}$ siècles alors que la documentation consultée mentionnait l'existence d'un orphelinat dès la fin du XVIII ${ }^{e}$ siècle. Le responsable des Archives forales de Biscaye nous a alors informée de la volonté du département des affaires sociales de cette province de maintenir secret l'ensemble des fonds ayant trait à l'abandon des enfants. 
17. Signalons néanmoins que les actuelles provinces de Cantabrie et de Guipúzcoa ont à l'inverse mis en place un système de catalogage qui facilite l'accès à ce type de documentation pour les périodes antérieures à 1845 .

18. La Ley del Patrimonio Histórico Español. province. Nous les retrouvons en Navarre et dans la province basque d'Alava ${ }^{17}$. Elles sont néanmoins contraires à la loi du 25 juin 1985 qui ouvre l'accès de l'ensemble des archives espagnoles après un délai de 50 ans $^{18}$. Pour justifier ce non-respect de la législation, les autorités biscayennes invoquent " des questions de droit au respect et à l'intimité des personnes ». Concernant une documentation vieille de plus de deux siècles, ce type de justification est peu convaincant. L'absence de rupture en matière de gestion documentaire lors du passage de la dictature franquiste à la démocratie et l'inertie des responsables locaux sont sans doute en partie à l'origine de cette politique très restrictive. Mais ne s'agit-il pas également de jeter un voile de discrétion sur des manquements institutionnels? Nous verrons que la lecture des fonds consultés incite à le croire.

Prenant appui sur les fonds de l'orphelinat provincial, cette recherche s'articule autour de quatre grandes thématiques. Après une présentation de la progressive et difficile prise en charge des orphelins de Biscaye, nous nous centrons sur l'inexorable progression des abandons. Par la suite, nous abordons les conditions d'assistance d'une institution au bord de la banqueroute. Les funestes conséquences d'une politique d'accueil discriminatoire, notamment en matière d'exposition des nourrissons, constituent notre dernier développement.

\section{LA CRÉATION DES PREMIÈrES MAISONS D'ACCUEIL DE BISCAYE}

En Biscaye, il n'existe aucune institution destinée à recueillir les enfants abandonnés jusqu’à la fin du $\mathrm{XVIII}^{\mathrm{e}}$ siècle. Au Moyen Âge, ils sont d'ordinaire pris 
en charge par leur famille, élevés par les seigneurs ou livrés à un sort funeste. À partir du Xv $\mathrm{xv}^{\mathrm{e}}$ siècle, les orphelins de la province sont confiés à l'hôpital de Saragosse ${ }^{19}$. Fondée en 1425 par Alphonse V, cette institution religieuse porte secours aux malades, aux pauvres et aux marginaux d'Aragon. Ses portes sont également ouvertes à tous les orphelins d'Aragon, de Navarre, de la Rioja et du Pays basque espagnol ${ }^{20}$. Notre Dame de Grâce de Saragosse couvre une région de plus de $70000 \mathrm{~km}^{2}$ et recueille entre 400 et 600 pupilles par an, dès le XVII ${ }^{\mathrm{e}}$ siècle $^{21}$. Entre 1781 et 1787 , la moyenne annuelle d'entrées s'élève à $875^{22}$.

Acheminés par convois, les délaissés de Biscaye parcourent, pour se rendre à Saragosse, une distance de plus de trois cent cinquante kilomètres dans des conditions exécrables. Sous-alimentés, maltraités et transportés en toute saison par lot de quatre ou de six, "tels de petits cochons de lait ${ }^{23}$ ", la plupart périssent avant la fin du périple ${ }^{24}$. Ces conditions de prise en charge ne sont néanmoins pas spécifiques à cette province. Nous les retrouvons aux quatre coins du royaume d'Espagne: à Valence, à Grenade, en Catalogne ${ }^{25}$ et en Galice où $90 \%$ des enfants trouvés périssent sur le chemin qui les mène à une maison d'assistance ${ }^{26}$. Notons que le devenir des orphelins français n'est guère plus enviable. Jusqu'au XvIII ${ }^{\mathrm{e}}$ siècle, quelle que soit la région considérée, toutes les recherches menées sur le sujet évoquent des nourrissons amoncelés dans des charrettes ou transportés dans des paniers par des meneurs qui les exposent aux intempéries. Dans ces conditions, très rares sont les survivants. Antoinette Chamoux ${ }^{27}$, Claude Delasselle ${ }^{28}$ et François Langlois ${ }^{29}$ s'accordent à penser que $90 \%$

19. El Hospital Real y

General de Nuestra Señora de Gracia de Zaragoza.

20. Sur le sujet, consulter l'article suivant: FERNÁNDEZ DOCTOR Asunción, MARTíNEZ VIDAL Álvaro, « El médico en el Hospital de Nuestra Señora de Gracia de Zaragoza en el siglo XVIII ", Dynamis: Acta Hispanica ad Medicinae Scientiarumque Historiam Illustrandam, volumen 5, 1985, p. 144.

21. AusÉns José Antonio, «De l'abandon à l'insertion sociale: les enfants trouvés de l'hôpital de Notre-DameDe-Grâce de Saragosse aux XVIII"-XIX ${ }^{e}$ siècles ", Noms et destins des sans famille, Paris, Presses de l'université Paris-Sorbonne, 2007 p. 321; MAISO GONZÁLEZ Jesús, «Aspectos del Hospital de Gracia y del Aragón bajo los Austrias ", Estudios, Saragosse, 1978, p. 302.

22. MaIso GonzÁLEZ Jesús, El hospital real y general de Nuestra Señora de Gracia de Zaragoza en el siglo XVIII, Saragosse, Estudios locales y comarcales, 2000, p. 301 et p. 309 .

23. VALVERDE LAMSFUS Lola, " Los niños expósitos guipuzcoanos en la inclusa de Pamplona en el siglo XVIII ", Bilduma Rentería 1, 1987, p. 112.

24. Sur les 394 orphelins transférés à Saragosse entre janvier et septembre 1792 seuls 92 ont survécu, soit un taux de mortalité de $76 \%$. Tout à fait plausible, ce pourcentage est encore largement inférieur aux chiffres avancés par Claude Delasselle qui estime que $90 \%$ des nourrissons transportés vers la capitale française décèdent avant l'âge de trois mois.

Delasselle Claude, « Les enfants abandonnés à Paris au xVIII' siècle ", Annales.

Économies, Sociétés, Civilisations, $n^{\circ} 1,1975$, p. 193.

25. Del Prado de la Fuente GaLÁN María, «La situación de las inclusas en el siglo XVIII. La encuesta de 1790 ", Chronica Nova, 24 1997, p. 65-74.

26. Pérez Moreda Vicente, "Las circunstancias del abandono ", Enfance abandonnée et société en Europe, XIV-XIXe siècle, Rome, 1991, p. 80.

27. Chamoux Antoinette, "L'enfance abandonnée à Reims à la fin du xvIII" siècle ", Annales de démographie historique, 1973, p. 264.

28. Delasselle Claude, « Les enfants abandonnés à Paris... ", op. cit., p. 193.

29. LANGLoIs François, "Les enfants abandonnés à Caen, 1661-1820 », Histoire, économie et société, 1987, $n^{\circ} 3$, p. 309 
30. La Maison des Enfants trouvés de Calahorra ou Casa de Expósitos de Calahorra.

31. Archivo Foral de Bizkaia (AFB), fondo expósitos de Bizkaia, registro 2, $14 / 03 / 1817$.

32. Cette décision royale va dans le sens de la législation française de janvier 1779 qui oblige toutes les villes du royaume à prêter assistance aux orphelins trouvés sur leur territoire. DeLASSELLE Claude, « Les enfants abandonnés à Paris... », op. cit., p. 216.

33. AFB, fondo expósitos de Bizkaia, registro 252, reglamento de 1845 .

34. Ibid., 1817.

35. AFB, fondo expósitos de Bizkaia, registro 15, 31/05/1799.

36. La Casa de Misericordia de Bilbao.

37. Les médecins de Calahorra justifient l'importante mortalité des petits Biscayens en précisant que la plupart franchissent les portes de l'institution le corps endommagé par de difficiles conditions de voyage. AFB, fondo expósitos de Bizkaia, registro 252 , Vizcaya. des orphelins transférés vers la région parisienne périssent avant d'arriver à destination.

En Biscaye, pour limiter l'hécatombe et favoriser la survie des enfants abandonnés, une seconde institution est créée en 1794 dans le diocèse de Calahorra ${ }^{30}$, circonscription dont dépendent cette province et une grande partie du Pays basque espagnol ${ }^{31}$. Destinée à l'accueil exclusif des enfants abandonnés, cette nouvelle maison représente un progrès dans la prise en charge de l'enfance délaissée de la région. Située dans l'actuelle province de la Rioja, elle ne permet toutefois pas de réduire la mortalité liée au transport des enfants. Alerté par des autorités diocésaines, Charles IV établit, par un décret de 1796, que seuls les enfants de plus de trois ans seront envoyés à Calahorra. Les plus jeunes seront pris en charge à proximité du lieu d'abandon par des nourrices rémunérées par l'évêché de Calahorra ${ }^{32}$. Mais cette circonscription ne peut très rapidement plus faire face à l'explosion du nombre des abandon $s^{33}$. Deux ans après sa création, deux filiales sont fondées à Mondragón et à Bilba ${ }^{34}$. Une troisième filiale a déjà été ouverte à Vitoria (province d'Alava) en 1796. Les autorités ecclésiastiques de chacune des trois provinces basques ont dorénavant l'obligation de s'occuper des orphelins de leur juridiction ${ }^{35}$.

C'est dans ce contexte que la Maison de la Miséricorde de Bilbao ${ }^{36}$ est choisie en 1798 pour accueillir les enfants abandonnés de la province. Mais cette nouvelle création ne résout pas totalement le problème du transport, les orphelins étant acheminés dans leur septième année vers la maison mère de Calahorra. Or, les conditions de voyage entre la Biscaye et Calahorra sont, à la fin du XVIII ${ }^{e}$ siècle, encore trop pénibles pour nombre de ces enfants ${ }^{37}$.

Pour éviter définitivement un transfert vers la Rioja, les autorités provinciales de Biscaye proposent au diocèse de Calahorra la création à Bilbao d'un nouvel hospice qui se chargera d'accueillir, de soigner et d'éduquer les orphelins de la province jusqu'à leur complète autonomie. Cet établissement - la Maison des Enfants trouvés de Biscaye ${ }^{38}$ - est créé en 1806. Fruit du récent intérêt de la monarchie espagnole pour l'enfance délaissée, cette nouvelle institution est exclusivement dédiée à l'assistance des enfants abandonnés. Totalement autonome et indépendante, elle ne dépend plus de l'évêché mais passe sous le contrôle de la province, la Diputación de Vizcaya.

Voyons maintenant comment les autorités politiques, par souci de mieux protéger les enfants délaissés, en sont venues, dans une conjoncture politique 
et économique extrêmement difficile, à favoriser l'abandon et la stigmatisation des nouveau-nés illégitimes.

\section{UNE INEXORABLE PROGRESSION}

\section{DES ABANDONS}

Jusqu'à la fin du XVIII ${ }^{\mathrm{e}}$ siècle, aucune source ne permet d'évaluer avec précision l'importance des délaissements d'enfants en Biscaye. En l'absence de maison d'accueil, il n'y a pas de lieu de prise en charge et par conséquent pas de statistiques. Les fonds ecclésiastiques tendent toutefois à prouver que l'abandon d'enfant n'est pas un comportement très répandu dans la Biscaye des $\mathrm{XVII}^{\mathrm{e}}$ et XVIII ${ }^{\mathrm{e}}$ siècles. À Portugalete ${ }^{39}$, aucun des 1331 enfants baptisés entre 1672 et 1700 n’a fait l'objet d'un délaissement. Au siècle suivant, nous n'en avons dénombré que deux sur 3493 naissances, soit un pourcentage de 0,06 \% ${ }^{40}$. Dans son étude sur la Biscaye orientale du XviII ${ }^{\mathrm{e}}$ siècle, Arturo Rafael Ortega Berruguete obtient des taux d'exposition systématiquement inférieurs à $0,4 \%{ }^{41}$. Dans la proche province de Guipúzcoa, on dénombre pour la période 1560-1659 6 enfants abandonnés pour 6681 baptêmes $(0,09 \%)^{42}$. En Navarre, à l'exception de la période 1660-1669, les proportions obtenues se maintiennent jusqu'en 1789 au-dessous des $0,5 \%{ }^{43}$. Selon Lola Valverde Lamsfus, ces pourcentages suggèrent que l'exposition des nouveau-nés est une pratique quasiment inconnue dans le Pays basque traditionnel ${ }^{44}$. Les données que nous avons recueillies imposent toutefois une certaine prudence: pour la période 17581779 , les fonds municipaux de Biscaye conservent

39. Située dans la banlieue de Bilbao, Portugalete devient dès le XVIII siècle l'une des grandes métropoles de la région.

40. Hanicot-Bourdier Sylvie, Portugalete: étude socio-démographique au XVIII siècle, inédit, p. 20.

41. Ortega Berruguete Arturo Rafael, « Un modelo de población socialmente autofrenado: la Vizcaya Oriental en el siglo XVIII », Ernoroa. Revista de Historia de Euskal Herria, n 3, 1986 p. 62.

42. VALVERDE LAMSFus Lola, Entre el deshonor y la miseria, infancia abandonada en Guipúzcoa y Navarra. Siglos XVIII y XIX, Bilbao, Universidad del País Vasco, 1994, p. 102-103.

43. Ibid., p. 121.

44. Ibid., p. 120. 
45. AFB, sección municipal, fondos municipales de Bilbao et de Begoña.

46. Cortés Cortés Fernando, «Mortalidad expósita en el Badajoz del seiscien-

tos », Enfance abandonnée et société en Europe..., op. cit., p. 442.

47. EGIDo Teófanes, «Aportación al estudio de la Demografía española: los niños expósitos de Valladolid (siglos XVIIXVIII) ", Actas de las I Jornadas de Metodología Aplicada de las Ciencias Históricas, volumen 3 , Santiago de Compostela, 1975 , p. 337.

48. Chacón JimÉnez Francisco, El GarRISTA Domeque Rosa, Fresneda Collado Rafael, « ¿ Mito o realidad? Análisis del comportamiento de las amas de cría en el reino de Murcia (siglos XVII-XVII) », Enfance abandonnée et société en Europe..., op. cit., p. 414.

49. VALVERDE LAMSFUS Lola, Entre el deshonor y la miseria, infancia..., op. cit., p. 16.

50. HANICOT-BOURDIER Sylvie, «Femmes et délits sexuels dans la province de Biscaye: le cas des ruptures de promesses de mariage ", Réalités et représentations du corps dans l'Europe, Nancy, Europe XVI-XVII, $n^{\circ} 16,2011$, p. 36

51. Hanicot-Bourdier Sylvie, "Le délit de concubinage dans la Biscaye du $\mathrm{xV} \|^{e}$ siècle », Réalités et représentations de la justice dans l'Europe des Xvi et $x v I^{\ominus}$ siècles, Nancy, Europe XVI-XVII, n० 17,2012 , p. 212-213
272 contrats d'adoption d'enfants abandonnés, soit une moyenne de douze contrats annuels ${ }^{45}$. Si ce nombre moyen semble peu important, il indique néanmoins que l'abandon des enfants n'est pas une pratique sociale totalement ignorée des Biscayens du XVIII ${ }^{\mathrm{e}}$ siècle. D'autre part, tous les orphelins n'ont pas la chance d'être adoptés. Il s'agit par conséquent d'une estimation a minima de la réalité du phénomène.

Il n'en demeure pas moins que les taux d'exposition obtenus sont nettement inférieurs à ceux enregistrés dans d'autres régions espagnoles. À Badajoz, Fernando Cortés Cortés recense de 1640-1699 un taux d'exposition de 5,8 \% ${ }^{46}$. 4104 enfants sont découverts dans les rues de Valladolid entre 1606 et $1659^{47}$. À Murcie, on accueille 3292 orphelins de 1650 à $1721^{48}$. Entre 1613 et 1659, l'orphelinat de Séville apporte protection à près de 10000 enfants.

Certaines caractéristiques de la famille basque traditionnelle seraient à l'origine de ces disparités. Au Pays basque, un système d'organisation sociale fondé sur l'existence de familles élargies aurait facilité l'intégration des enfants illégitimes et limité le nombre des expositions ${ }^{49}$. L'étude des archives notariales incite à penser que les relations pré-matrimoniales, sous couvert de promesse de mariage, constituent un phénomène relativement fréquent dans la Biscaye du XVII ${ }^{\mathrm{e}}$ siècle $^{50}$. À la même époque, les témoignages recueillis dans les procès pour faits de concubinage révèlent la tolérance d'une communauté villageoise qui ne se scandalise pas systématiquement de l'existence de cohabitations pré-matrimoniales entre jeunes gens qui ont promis de s'épouser ${ }^{51}$. À ce sujet, 
l'analyse des registres paroissiaux de la commune de Portugalete indique que $20,3 \%$ des couples qui se marient entre 1701 et 1800 ont déjà une fille ou un fils dit "légitime ${ }^{52}$ ". Dans la Biscaye orientale du XviII siècle, Arturo Rafael Ortega Berruguete signale également que les prêtres basques inscrivent dans leurs registres comme légitimes de nombreux enfants naturels issus de parents connus et dont le futur mariage ne fait aucun doute, ce qui plaide en faveur de l'existence de cohabitations pré-matrimoniales ${ }^{53}$. Dans son étude sur la société française traditionnelle, Jean-Louis Flandrin rappelle que le concubinage est parfois une nécessité pour des individus pas assez riches pour supporter les frais d'une union légale. Aussi, aux XVIII ${ }^{\mathrm{e}}$ et $\mathrm{XIX}^{\mathrm{e}}$ siècles, les unions libres se répandent-elles dans les milieux urbains les plus modestes. Les valeurs observées sont toutefois bien au-dessous de celles enregistrées dans notre province. À titre d'exemple, dans le Cambrésis du XviII ${ }^{\mathrm{e}}$ siècle, moins de $2 \%$ des femmes qui introduisent devant la justice une action en reconnaissance de paternité avouent une relation durable en dehors des liens du mariage ${ }^{54}$.

Jusqu'à la fin du XviıI ${ }^{\mathrm{e}}$ siècle, l'abandon d'enfant constitue un acte exceptionnel dans bien d'autres régions. Cette réalité se vérifie dans la Galice rurale, l'actuelle province de Léon, mais aussi au sud de la péninsule, en Estrémadure ${ }^{55}$. La présence d'un système d'assistance aux enfants abandonnés et la mise en place de règles d'accueil plus ou moins restrictives ont par la suite un effet considérable sur l'évolution numérique des abandons. Cette incidence se retrouve à Léon, à Saint-Jacques-de-Compostelle, à Pampelune, à Séville ${ }^{56}$, où l'ouverture d'une maison d'accueil génère une importante progression des délaissements.

En Biscaye, les registres de la Casa de Misericordia de Bilbao, puis de la Casa de Expósitos de Vizcaya, qui permettent de suivre l'évolution de la courbe des abandons d'enfants à partir de 1798, témoignent d'une spectaculaire augmentation de l'exposition (Graphique 1). Marginal à la fin du XVIII ${ }^{\mathrm{e}}$ siècle, l'abandon de nourrisson devient une pratique habituelle dans les années 1830. En l'espace de 42 ans, la moyenne annuelle passe de 7,3 cas pour la période 1798-1800 à 163,2 entre 1836 et 1840 , soit une multiplication par 22,4. À l'exception du quinquennat 1816-1820, qui enregistre une baisse non significative car uniquement attribuable à l'importante progression de la période précédente, l'augmentation est continue par période de cinq ans.

Plusieurs facteurs concomitants ont contribué à cette explosion. Signalons tout d'abord un probable sous-enregistrement des délaissements à la fin du

\author{
52. Hanicot-Bourdier Sylvie, \\ Portugalete aux XVIII et \\ $x \mid x^{e}$ siècles: contribution à \\ une étude socio-démogra- \\ phique, Villeneuve d'Ascq, \\ Presses universitaires \\ du Septentrion, 2003, \\ p. 301-302. \\ 53. Peu étudiée, cette \\ pratique se trouve à \\ Portugalete, mais également \\ dans la Biscaye orientale \\ Hanicot-Bourdier Sylvie, \\ Portugalete..., op. cit., \\ p. 299; Ortega Berruguete \\ Arturo Rafael, « Un modelo \\ de población socialmente \\ autofrenada... ", op. cit., \\ p. 61-62. \\ 54. Minvielle Stéphane, La \\ famille en France à l'époque \\ moderne: XVI'-XVIII siècle, \\ Paris, Armand Colin, 2010, \\ p. 127-128. \\ 55. Dubert Isidro, \\ "L'abandon d'enfants \\ dans l'Espagne de l'Ancien \\ Régime: réévaluer l'ampleur \\ et les causes du phéno- \\ mène ", Annales de démo- \\ graphique historiques, n 125, \\ 2013, p. 145.
}

56. Ibid., p. 151-153. 


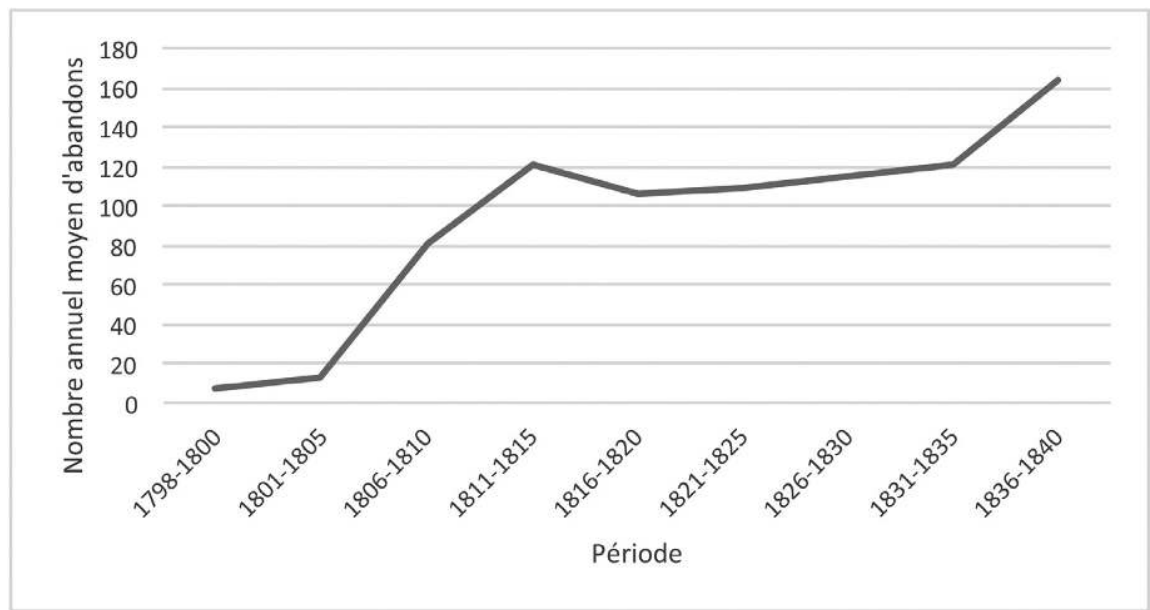

Graphique 1: Nombre annuel moyen d'abandons à la Casa de Misericordia de Bilbao et à la Casa de Expósitos de Vizcaya (1798-1840).

Sources: Archivo Foral de Bizkaia, fondo expósitos de Bizkaia, registro 15, registro 252.

57. Dans les premières années de fonctionnement de la Casa de Misericordia de Bilbao, il est pratiquement sûr qu'un certain nombre d'abandons échappent à cette institution et sont encore directement pris en charge par les autori-

tés locales.

58. Les communes les plus dynamiques enregistrent un taux de croissance annuel supérieur à 2,5 \% entre 1800

et 1824. HANICOT-BOURDIER

Sylvie, Portugalete... op. cit., p. 34

59. VALVERDE LAMSFUS Lola, Entre el deshonor y la miseria, infancia..., op. cit., p. 65 ; Gracia CÁRCAMO Juan, « La otra sociedad: los marginados », Revista Internacional de Estudios Vascos, n41-42, Donostia, 1996, p. 531.
$\mathrm{XVIII}^{\mathrm{e}}$ siècle et au début du siècle suivant ${ }^{57}$. La seconde explication est l'augmentation de la population. Rappelons que la Biscaye connaît dès le début du XIX ${ }^{\mathrm{e}}$ siècle une importante croissance démographique et urbaine ${ }^{58}$. Mais ces explications sont largement insuffisantes. Au tout début de ce siècle, le nombre de délaissements est pratiquement multiplié par deux en l'espace de cinq années: on enregistre une moyenne de 7,3 abandons par an de 1799 à 1800 , puis de 13,2 en 1801-1805 (+ 78,7\%). Ce dernier quinquennat correspond à une période catastrophique dans une grande partie de la péninsule Ibérique. Partout, la perte de récoltes et des famines poussent de nombreuses familles miséreuses à se défaire de leurs nourrissons ${ }^{59}$. La progression des abandons est également imputable aux dispositions royales qui, en s'intéressant à la détresse des petits exposés, ont renforcé un problème qu'elles prétendaient résoudre. En 
interdisant aux autorités judiciaires d'arrêter ou d'interroger toute personne qui affirme avoir l'intention de déposer un enfant dans une maison d'accueil ou entre les mains d'un prêtre, il y a tout lieu de penser que l'article 23 de l'ordonnance royale du 11 décembre 1796 contribue à stimuler les délaissements. Ce décret banalise également l'exposition sauvage en garantissant l'indulgence de la justice à toute personne qui, ayant exposé un enfant, prévient suffisamment tôt les autorités ecclésiastiques du lieu d'abandon ${ }^{60}$. En Biscaye, les effets incitatifs de cette nouvelle législation se combinent, à partir de 1798, avec ceux de l'ouverture des premières institutions d'accueil. Ainsi, après la fondation en 1806 de la Casa de Expósitos de Vizcaya, le nombre des abandons d'enfants connaît à nouveau une spectaculaire progression passant de 15 en 1806 à 92 en 1807 , puis à 88 en 1808.

Ces évolutions du début du XIX ${ }^{e}$ siècle correspondent à celles repérées en Navarre ${ }^{61}$, mais également en Galice ${ }^{62}$ et dans l'actuelle province de Cantabrie $^{63}$. Dans ces régions du Nord-ouest de la péninsule, l'ouverture de nouveaux hôpitaux destinés à secourir les orphelins, associée à un appauvrissement de la population, aurait généré une modification des comportements familiaux. Pouvant compter sur l'accueil institutionnel de leur nourrisson, les mères célibataires ou les familles miséreuses seraient davantage enclines à s'en défaire ${ }^{64}$. Mais l'installation d'une maison d'assistance a aussi révélé une réalité sociale jusqu’alors restée cachée.

De 1809 à 1813 , pendant la guerre d'indépendance contre Napoléon, le nombre annuel des
60. Serrano Ruiz-Calderón Manuel, «El abandono de menores: su regulación en el ámbito penal », Revista del Ministerio de Trabajo $y$ asuntos sociales, $\mathrm{n}^{\circ} 45$, 2003, p. 35

61. VALVERDE LAMSFUs Lola, Entre el deshonor y la miseria, infancia..., op. cit., p. 59

62. Dubert Isidro,

« L'abandon d'enfants... », op. cit., p. 158 .

63. LANZA García Ramón, La población y el crecimiento económico de Cantabria en el Antiguo Régimen, Madrid Universidad Autónoma de Madrid-Universidad de Cantabria, 1991, p. 301.

64. Mentionnons deux exemples. À Santander, les enfants trouvés ne représentent que $0,4 \%$ des baptisés avant 1780. Après la fondation d'une institution d'accueil, cette proportion atteint 8,5\%. Dans la commune galicienne de Ferrol, un hospice est créé en 1786. Entre les périodes 1752-1755 et 1795-1797, le taux d'exposition passe de 1,2 à 16,7 \%. LANZA GARcía Ramón, La población.... op. cit., p. 301 ; DUBERT Isidro, « L'abandon d'enfants... », op. cit., p. 158. 
65. García Merino Luis Vicente, La formación de una ciudad industrial. El despegue urbano de Bilbao, Bilbao, Instituto Vasco de Administración pública, 1987, p. 353; GracIA CÁrcamo J., Mendigos y vagabundos en Vizcaya (1766-1833), Bilbao, Universidad del País Vasco, p. 117.

66. URQUIJO y GoITIA José Ramón, « los sitios de Bilbao », Vasconia: Cuadernos de historiageografía, n¹0, 1988, p. 9-36.

67. Madoz Pascual, Diccionario geográfico-estadístico-histórico de España y sus posesiones de Ultramar, tomo IV, Madrid, Imp. del Diccionario GeográficoEstadístico-Histórico de D. Pascual Madoz, 1849, p. 321 . abandons se maintient entre 99 et 123. L'impact de ce conflit se révèle également crucial une fois les hostilités terminées. La Biscaye - dont la population et l'économie sont totalement dévastées - enregistre des niveaux de délaissement encore inconnus jusqu'alors. On recense sur cette période une multiplication par 1,4 du nombre des abandons: 149 enfants sont rejetés par leurs parents en 1814; ils étaient 104 en 1813 et 114 en 1812.

Après un léger répit entre 1816 et 1820 , les abandons repartent inexorablement à la hausse. Pour 1816-1820, la moyenne quinquennale est de 105,8 cas par an, elle s'élève à 109,4 et à 114,2 pour les périodes $1821-1825$ et 18261830. Cette croissance coïncide avec une dépression économique associée à une augmentation de la pauvreté ${ }^{65}$. Mais la période la plus critique reste encore à venir.

C'est entre 1836 et 1840 que les chiffres de l'abandon atteignent leur plus haut niveau, puisque au cours de cette période la Casa de Expósitos de Vizcaya reçoit en moyenne 163,2 nouveaux pensionnaires par an. La situation la plus grave est enregistrée en 1838 et 1839 où 171 et 188 abandons sont répertoriés dans les livres de l'institution. Rappelons qu'entre 1833 et 1839, la Biscaye et sa capitale Bilbao - plusieurs fois assiégée $e^{66}$ - subissent de plein fouet les effets néfastes de la première guerre carliste. Selon Pascual Madoz, ce conflit, associé à la démoralisation et à l'appauvrissement de la population, serait à l'origine d'une progression des abandons d'enfants dès $1833^{67}$. Mais il a également un impact direct sur la prise en charge des orphelins, les carlistes occupant l'ensemble de la région à l'exception de Bilbao qui demeure sous contrôle libéral. Deux autorités provinciales - la Diputación de Vizcaya à Bilbao et la Diputación carlista dans le reste de la province - gèrent l'accueil des enfants trouvés. Cette double compétence pose de réelles difficultés d'organisation. Les habitants de la région ne savent plus à qui s'adresser en cas d'exposition sur la voie publique. Citons le cas de quatre femmes qui, originaires d'Orduña, apportent quatre nouveaunés exposés à la Maison des Enfants abandonnés de Bilbao le 4 août 1839. Un seul a été baptisé par les autorités libérales. Les trois autres ont reçu le premier des saints sacrements de la main des carlistes, raison pour laquelle la direction de l'orphelinat refuse de les admettre. Celle-ci considère qu'ils auraient dû être conduits à Durango où les carlistes ont installé un établissement pour recueillir les enfants abandonnés sur le territoire qu'ils occupent. Heureusement pour les trois orphelins, la Diputación de Vizcaya ordonne finalement à l'orphelinat 
provincial de les prendre en charge, la ville d'Orduña étant récemment passée sous contrôle libéral ${ }^{68}$. Ces difficultés de fonctionnement se retrouvent dans les fonds consultés. Pratiquement inexistants dans les années antérieures, les désordres chronologiques sont nombreux entre 1833 et 1839 . Certains pupilles attendent plus de cinq années avant de voir leur acte de baptême consigné dans les registres de l'institution censée les prendre en charge. On imagine aisément que les urgences de la guerre, le blocage des routes et le siège de Bilbao sont à l'origine de ces irrégularités. Dans ces conditions, tout laisse à penser que nos estimations de la fréquence des expositions sont, pour la période 1833-1839, en-dessous de la réalité.

Nos recherches antérieures tendent à prouver qu'un changement des mentalités a aussi contribué à accroître le nombre des abandons. À Portugalete, l'examen parallèle de l'abandon d'enfants et des naissances hors mariage révèle que la période 1826-1850 voit coïncider le plus fort taux moyen d'exposition $(2,3 \%)$ et le plus faible taux moyen d'illégitimité $(0,6 \%)^{69}$. Une situation identique se produit dans la proche province de Navarre, où ne plus baptiser son enfant dans sa paroisse d'origine mais le confier directement à une institution devient clairement un moyen de cacher une conception hors mariage $\mathrm{e}^{70}$. L'explication vaut-elle pour la Biscaye? Une réprobation morale plus sévère vis-à-vis des filles-mères aurait-elle conduit certaines d'entre elles à abandonner l'enfant naturel qu'elles déclaraient et élevaient quelques décennies auparavant? Plusieurs indices plaident en faveur de cette hypothèse et semblent témoigner à la fois d'un opprobre grandissant à leur égard et d'une dégradation de leurs conditions de vie. L'augmentation au XVIII ${ }^{\mathrm{e}}$ siècle des actes de procédure pour fait de concubinage signale un indéniable durcissement des mœurs ${ }^{71}$. L'étude des fonds judiciaires et des sources notariales révèle quant à elle la plus grande difficulté des jeunes mères célibataires biscayennes du XVIII ${ }^{\mathrm{e}}$ siècle à obtenir une compensation en cas de rupture d'engagement matrimonial ${ }^{72}$. Là encore, on observe la même situation en Navarre ${ }^{73}$.

\section{UNE INSTITUTION AU BORD DE LA BANQUEROUTE}

Dès 1774, les administrateurs de la Casa de Misericordia de Bilbao s'inquiètent des difficultés financières de l'établissement. Ils doivent affronter une recrudescence de la pauvreté et indiquent être dans l'obligation de contrôler l'origine géographique des miséreux qui leur demandent asile. Seules les

\author{
68. AFB, fondo expósitos \\ de Bizkaia, registro 2 , \\ 06/08/1839. \\ 69. Hanicot-Bourdier Sylvie,
Portugalete..., op. cit., \\ p. 264. \\ 70. VALVERDE LAMSFUS Lola, \\ Entre el deshonor y la mise- \\ ria, infancia..., op. cit., p. 78. \\ 71. HANICOT-BOURDIER \\ Sylvie, « Le délit de \\ concubinage... », op. cit., \\ p. 210-211. \\ 72. HANICOT-BOURDIER \\ Sylvie, «Femmes et délits \\ sexuels... ", op. cit., \\ p. 36-38. \\ 73. VALVERDE LAMSFUS Lola, \\ Entre el deshonor y la \\ miseria, infancia..., op. cit., \\ p. 80-81.
}


74. AFB, sección municipal, fondo archivo municipal de Bilbao, asignatura Bilbao Antigua 0318/001/032, 28/02/1774

75. AFB, fondo expósitos de Bizkaia, registro 2, $14 / 03 / 1817$

76. Ibid.

77. Ibid.

78. AFB, fondo expósitos de Bizkaia, registro 2, 31/12/1837.

79. AFB, sección municipal, fondo archivo municipal de Bilbao, asignatura Bilbao Antigua 0329/001/029/001, 29/10/1807.

80. AFB, sección municipal, fondo archivo municipal de Bilbao, asignatura Bilbao Antigua 0355/001/084, 14/11/1824.

81. AFB, fondo expósitos de Bizkaia, registro 252, 14/06/1812.

82. AFB, fondo expósitos de Bizkaia, registro 2, 28/06/1812. personnes originaires de Bilbao ou ayant travaillé au moins dix années dans cette commune reçoivent désormais l'asile et l'aide demandés. Pour éviter qu'ils n’affluent dans la province, les étrangers sont moins bien traités et ne reçoivent qu'un seul repas, le déjeuner ou le dîner. Après une nuit de repos et un petit déjeuner, ils sont invités à quitter rapidement la région ${ }^{74}$. Vingt ans plus tard, le contexte financier ne s'est pas amélioré, et la création, en 1794, d'une filiale de la Casa de Expósitos de Calahorra au sein d'une institution déjà fortement endettée constitue une gageure. Quelques années à peine après sa fondation, cet orphelinat est dans l'impossibilité de rémunérer les services des nourrices qui accueillent les enfants de moins de trois ans. Faute de fonds suffisants, nombre d'orphelins sont - malgré leur très jeune âge - envoyés à Calahorra ${ }^{75}$. Au fil du temps, les difficultés budgétaires ne cessent de s'aggraver.

Dans l'histoire de l'institution, 1806 constitue une année charnière. En passant sous la tutelle des autorités provinciales, la Maison des Enfants abandonnés de Biscaye perd une source de revenus importante, le diocèse de Calahorra cessant de la subventionner ${ }^{76}$. Pour pourvoir à l'alimentation et à l'entretien de ses pensionnaires, elle doit dorénavant se contenter des sommes payées par les Biscayens qui souhaitent échapper au jeûne quadragésimal ${ }^{77}$. À peine quelques semaines après sa prise de fonction, son conseil d'administration - composé de notables issus du secteur privé - écrit aux évêques de Santander et de Calahorra pour leur demander de prendre l'établissement sous leur protection ${ }^{78}$.

Surendettée, la Casa de Expósitos de Vizcaya doit fréquemment faire appel à la générosité privée. Dès octobre 1807, soit quelques mois après son passage sous la coupe des autorités civiles, elle implore les vicaires et les prêtres de la région de rappeler à leurs paroissiens la nécessité de participer à l'entretien de l'établissement. Pour inciter la population à la charité, elle suggère d'évoquer les charges croissantes de l'orphelinat ou toute autre raison jugée appropriée ${ }^{79}$. La même situation se réitère en novembre $1824^{80}$.

Le 14 juin 1812, l'orphelinat exhorte les Biscayens à racheter leurs péchés en secourant les enfants trouvés de la province qui, pauvres parmi les plus pauvres, ne peuvent que susciter la pitiei ${ }^{81}$. Centré sur une vision religieuse de la charité, ce discours se double d'une dimension économique et utilitaire. Le 28 juin 1812, le conseil d'administration de la Maison des Enfants abandonnés de Biscaye suggère que « la mort de ses pupilles prive la société du futur artisan, marin et paysan dont elle aura besoin ${ }^{82}$ ». Ce désir de veiller à l'avenir 
économique du pays se retrouve six jours auparavant dans un courrier de la direction de l'établissement qui se déclare satisfaite d'avoir sauvé de petits êtres désemparés qui, « de par leur robustesse, fournissent à l'agriculture et à l'artisanat les bras dont ils ont tellement besoin en ces lendemains de guerre et de reprise économique ${ }^{83} »$. Vingt et un ans plus tard, la province rappelle à son tour que les nourrissons secourus deviendront « des êtres utiles à la Nation ${ }^{84}$ ».

Mais face à la recrudescence de l'exposition, les appels à la compassion ne suffisent pas. Pour subvenir à une augmentation exponentielle des besoins, les autorités provinciales sollicitent très fréquemment les municipalités. La perception des fonds exigés est souvent problématique, les communes ayant déjà du mal à faire face à leurs propres dépenses. Le 22 juin 1812, le responsable provincial Manuel de Landaida s'étonne de ce manque de collaboration estimant que cette participation financière ne devrait pas être considérée comme " une nouvelle charge, mais plutôt comme une aumône, un don gratuit auxquels les localités devraient se soumettre avec joie». En ce sens, il rappelle que soulager les souffrances de « malheureux enfants " qui « implorent à cor et à cri un secours " constitue une obligation religieuse, mais aussi humanitaire et politique ${ }^{85}$.

Trois mois plus tard, la province menace les municipalités de recourir à la force en cas de non versement des sommes dues ${ }^{86}$. Malgré ces difficultés de recouvrement, la province établit un nouvel impôt en janvier 1816 sur l'ensemble des propriétés foncières qui sont taxées à hauteur de $3 \%$ de leur valeur. Les locataires des dits biens doivent s'acquitter du tiers de cette nouvelle contribution; le reste est à la charge des propriétaires ${ }^{87}$. La région se déclare consciente du nouveau sacrifice qu'elle impose à des Biscayens qui, anéantis et épuisés par d'innombrables vols, saccages et malheurs consécutifs à l'occupation française, n'ont souvent même pas de quoi subvenir à leurs propres besoins. Mais "l'infâme conduite " de l'occupant français et l'état déplorable de l'orphelinat provincial ne lui laisseraient aucun autre choix ${ }^{88}$.

Par manque de ressources, les autorités provinciales en charge de l'enfance délaissée ne respectent pas toujours leurs obligations vis-à-vis des municipalités. Citons plusieurs exemples. En pleine guerre d'indépendance contre Napoléon, le village de Zarátamo adresse un courrier à la Diputación le 18 avril 1814 . Il déplore que cette dernière n'ait pas tenu ses promesses et la menace de rendre l'enfant exposé qu'il a sauvé d'une mort certaine, s'il n'obtient pas une réponse

\author{
83. Ibid., 22/06/1814. \\ 84. AFB, fondo expósitos \\ de Bizkaia, registro 252, \\ 17/11/1833. \\ 85. Ibid., 22/06/1812. \\ 86. Ibid., 10/10/1812. \\ 87. Ibid., 11/01/1816. \\ 88. Ibid., 11/01/1816.
}


89. AFB, sección administración de Bizcaya, subsección gobierno y asuntos eclesiásticos, asignatura AJ01294/145, 18/04/1814.

90. Ibid.

91. AFB, sección administración de Bizcaya, subsección gobierno y asuntos eclesiásticos, asignatura AJ01201/008, 16/06/1814; fondo expósitos de Bizkaia, registro 2, 03/07/1817.

92. AFB, fondo seguridad pública, guerras y servicio militar, asignatura AQ01395/283, 31/08/1835.

93. AFB, sección administración de Bizcaya, subsección gobierno y asuntos eclesiásticos, asignatura AJ00210/029, 30/01/1816.

94. Ibid.

95. Jusqu'au xixe siècle, l'Alcalde est un juge avec une compétence civile et criminelle. Par la suite, ce terme désigne le maire d'une commune.

96. AFB, fondo expósitos de Bizkaia, registro 16, 12/07/1816. favorable à sa demande de remboursement. Un mois auparavant, une petite fille de quelques heures a été trouvée dans la nuit du 18 mars. La municipalité lui a fourni quelques vêtements et lui a rapidement trouvé une nourrice. Malgré les nombreux voyages de la mère de lait à la capitale, l'orphelinat n'a toujours pas pourvu à l'habillement de l'enfant. Pour rémunérer la nourrice, la commune de Zarátamo s'est engagée à lui verser une rémunération annuelle de 640 réaux. Les familles nourricières recrutées par l'institution ne gagnent qu'un réal par jour d'allaitement. Aussi la direction de l'établissement refuse-t-elle de payer le salaire promis par la municipalité ${ }^{89}$.

En 1814, les archives forales conservent la trace d'une seconde procédure: celle engagée par la ville de Galdames qui réclame le remboursement des 320 réaux dépensés pour nourrir une petite fille trouvée le 9 octobre de cette même année. Le 11 novembre 1814, elle essuie également une fin de non-recevoir au motif que la Casa de Expósitos de Vizcaya n'a pas été informée en temps et en heure de la découverte de l'enfant ${ }^{90}$.

Les soucis d'économie de l'institution se lisent également dans les différents courriers que son vice-économe adresse aux autorités locales. En 1814, ce dernier exige que les habits des orphelins décédés - même en très mauvais état - soient récupérés et lavés afin d'être réutilisés ${ }^{91}$. Les familles d'accueil qui seraient dans l'obligation d'acheter des vêtements pour les enfants dont elles ont la garde doivent se contenter du strict minimum, sans aucun surplus ${ }^{92}$.

Deux ans plus tard, la commune de Zaldívar se voit refuser en janvier 1816 le remboursement des 121 réaux qu'elle a déboursés pour habiller le petit Valentin, un bébé exposé le 13 février 1814. L'orphelinat considère cette dépense excessive au regard des 80 réaux qu'il a l'habitude de dépenser pour fournir quatre rechanges aux enfants qu'il confie à des familles nourricières ${ }^{93}$. N'ayant jamais été informé des soucis de santé de Valentin, il n'est pas non plus disposé à prendre en charge les 60 réaux de frais médicaux payés par la municipalité ${ }^{94}$.

Extrême, mais également significatif, est le cas d'un petit garçon trouvé de trois ans. Faute de moyens, sa nourrice ne peut pas avancer l'argent nécessaire pour le vêtir. Le dénuement de cet orphelin alerte l'Alcalde ${ }^{95}$ de Marquina qui exige que l'orphelinat provincial envoie quelques habits à un enfant qui «se promène pratiquement $\mathrm{nu}^{96}{ }^{9}$. 
Plusieurs villes rappellent à la Casa de Expósitos de Vizcaya son obligation de pourvoir à l'habillement des orphelins ${ }^{97}$. Mais d'autres, moins soucieuses du confort des nourrissons abandonnés et peu disposées à avancer des frais dont elles ne seraient sans doute pas remboursées, n’hésitent pas à envoyer les enfants trouvés à l'orphelinat, vêtus des rares guenilles dont ils étaient couverts lors de leur exposition ${ }^{98}$.

Parce qu'ils n'ont pas l'assurance d'être rémunérés, nombre de médecins refusent de vacciner les orphelins et de les assister en cas de maladie. Dans une circulaire, qu'elle envoie aux municipalités le 4 janvier 1831, la province dénonce cette non-assistance à l'enfance délaissée ${ }^{99}$. En 1845, elle tente de remédier à ce manque de conscience professionnelle - et d'alléger ses charges - en décrétant que les médecins municipaux ont dorénavant l'obligation d'assister gratuitement les orphelins qui auraient besoin de leurs services ${ }^{100}$.

Signe que les considérations financières l'emportent sur toutes les autres, obligation est faite aux municipalités de contrôler l'origine géographique des exposés qu'elles envoient à Bilbao. Dans un contexte matériel difficile, la province ne saurait prendre en charge des enfants nés dans une région limitrophe sans « léser " les petits Biscayens dont elle a la responsabilité. Dans un procès intenté contre une mère pour fait d'exposition, la Diputación de Vizcaya se plaint dès 1807 de l'augmentation des frais qu'elle doit engager suite à une recrudescence des expositions d'enfants originaires d'autres provinces ${ }^{101}$.

En 1812, les responsables de l'enfance délaissée biscayenne évoquent "l'introduction furtive" sur le sol biscayen de bébés originaires des proches provinces de Guipúzcoa, d'Alava et de Castille. Pour éviter les préjudices financiers que de telles pratiques occasionnent à la Maison des Enfants abandonnés de Biscaye, ils ordonnent aux prêtres de contrôler la provenance géographique des nouveau-nés abandonnés ${ }^{102}$.

\section{UNE POLITIQUE D'ACCUEIL DISCRIMINATOIRE}

\section{ET SES FUNESTES CONSÉQUENCES}

Fondée pour maintenir en vie des orphelins de plus en plus nombreux, la Casa de Expósitos de Vizcaya est victime de son succès. Entre 1806 et 1843, elle accueille 4496 pensionnaires ${ }^{103}$. En 1807 , à peine un an après sa création, ils

\author{
97. Ibid., 11/11/1814 et \\ $15 / 12 / 1814$. \\ 98. AFB, fondo expósitos \\ de Bizkaia, registro 15, \\ 25/01/1809 et 09/09/1811. \\ 99. AFB, fondo expósitos \\ de Bizkaia, registro 252, \\ 04/01/1831. \\ 100. Ibid., reglamento de \\ 1845. \\ 101. AFB, fondo corregi- \\ dor, subfondo criminal, \\ asignatura JCR4095/021, \\ 14/11/1807. \\ 102. AFB, fondo expósitos \\ de Bizkaia, registro 252, \\ 07/07/1812. \\ 103. Ibid., reglamento de \\ 1845.
}


104. Ibid., reglamento de 1845.

105. AFB, fondo expósitos de Bizkaia, registro 252, 14/10/1814, 22/10/1814.

106. AFB, fondo expósitos de Bizkaia, registro 2, 28/08/1816.

107. Ibid., 03/07/1817.

108. A.F.B, fondo expósitos de Bizkaia, registro 2, 03/07/1817. sont déjà 98 à bénéficier de sa prise en charge. En 1836, ils sont 713, soit une multiplication par $7 \mathrm{du}$ nombre de pupilles.

En proie à d'importantes difficultés financières dès l'année de sa fondation, l'établissement ne peut envisager d'augmenter le nombre de ses employés. En juillet 1806, il enregistre déjà un déficit de 159589 réaux ${ }^{104}$. Pendant la guerre contre les troupes d'occupation françaises, son solde débiteur atteint 226000 réaux en 1812 , puis s'élève à 243817 réaux en 1814 . En octobre de cette même année, ses administrateurs s'inquiètent sérieusement de l'accroissement quotidien du nombre d'orphelins. Les 460 enfants à leur charge sont, faute de ressources, "victimes d'une extrême indigence ${ }^{105}$ ». Totalement " anéanties ", les ressources de la Province ne permettent plus en 1816 de couvrir les besoins les plus élémentaires des orphelins de Biscaye ${ }^{106}$.

À force de courriers, la direction de l'établissement obtient plusieurs versements exceptionnels entre 1817 et 1829. Mais les fonds disponibles s'avèrent tout à fait insuffisants et ne permettent aucunement d'affronter la progression des dépenses qu'engendre la recrudescence de l'exposition dans les années 18201830. En l'espace de deux années, les dettes de la Casa de Expósitos progressent de $187,4 \%$ pour atteindre 355120 réaux en 1821. Mais le pire est encore à venir: en pleine guerre carliste, son déficit budgétaire atteint 879011 réaux en 1837, puis la somme colossale de 1037948 réaux en 1838.

Devant faire face à la croissance du nombre des admissions et à une pénurie chronique de moyens financiers, les administrateurs de la Casa de Expósitos de Vizcaya ont la volonté délibérée de restreindre leur accueil aux seuls enfants illégitimes ou trouvés. Appliquant le règlement à la lettre, ils refusent systématiquement de prendre à leur charge les nourrissons nés de parents légitimement mariés ${ }^{107}$.

Des raisons financières justifient en partie ce refus de prise en charge. Mais les autorités provinciales redoutent également un détournement de l'assistance par des couples mariés qui pourraient être tentés d'abandonner provisoirement leurs enfants afin de ne pas avoir à supporter les frais d'allaitement. Cette crainte se lit implicitement dans le règlement de la Maison des Enfants abandonnés de Biscaye qui interdit de communiquer à quiconque la destination ou l'identité des nourrices des enfants délaissés ${ }^{108}$. L'objectif d'une telle pratique est de décourager les couples pauvres d'abandonner leurs bébés. Dans cette perspective, l'orphelinat rappelle systématiquement aux couples légitimes pauvres, qui 
se voient refuser l'admission de leur enfant, qu'ils peuvent recevoir une aide de leur commune d'origine ${ }^{109}$. Cette dichotomie entre enfants légitimes et enfants naturels n'est pas spécifique à notre province. Dans le Turin du XviII ${ }^{\mathrm{e}}$ siècle, deux hôpitaux coexistent. Le premier est réservé à l'accueil exclusif des enfants nés dans le cadre d'une union consacrée, le second aux nourrissons nés hors mariage. À la même époque, à Porto, on accorde des subsides aux couples mariés pour qu'ils élèvent leurs nourrissons, et l'on canalise les dépôts anonymes des illégitimes vers la Casa de Roda. Cette pratique se retrouve à Rouen ${ }^{110}$. Mais, étonnamment les orphelinats des proches provinces de Guipúzcoa et de Navarre adoptent un mode de fonctionnement différent de celui de la Biscaye, et reçoivent entre leurs murs les enfants légitimes des parents les plus nécessiteux. L'objectif est de lutter contre l'exposition sauvage et les risques qu' elle fait peser sur la santé des bébés ${ }^{111}$.

En Biscaye, en cas de refus de soutien municipal, la politique d'accueil discriminatoire des autorités provinciales condamne les couples mariés à exposer anonymement les nourrissons qu'ils ne peuvent pas éduquer faute de moyens. Lorsqu'ils ont la chance de survivre - dans les procédures judiciaires intentées pour faits d'exposition en Biscaye, entre 1801 et 1820 , seuls $22 \%$ des bébés ont été découverts encore en vie -, ces bambins légitimes rejoignent alors la cohorte des enfants trouvés sur lesquels pèse une forte suspicion d'illégitimité et donc d'immoralité.

Malgré les risques encourus, l'exposition d'enfant demeure une pratique courante dans la Biscaye du début du XIX ${ }^{\mathrm{e}}$ siècle: entre 1807 et 1839 , près d'un nourrisson sur trois (1 173 bébés, soit $30 \%$ ) subit ce type d'abandon ${ }^{112}$. Oscillant entre $27,8 \%$ et $30,1 \%$ (soit un écart de $2,3 \%$ ), la proportion des nourrissons trouvés reste stable jusqu'en 1830. Par la suite, la période 18311839 enregistre une augmentation relativement importante des abandons sauvages, de l'ordre de $5 \%$. Cette progression s'accompagne d'une baisse des présentations d'enfants qui passent de $11,8 \%$ à $6,3 \%$. Encore plus marquées de 1833 à 1839, ces évolutions sont imputables à la première guerre carliste qui modifie les modalités de délaissement des nourrissons: pendant cette période, seuls 4,9\% des abandonnés sont amenés jusqu'à un prêtre. Dans les trois dernières années du conflit, cette proportion tombe à 3,3\%. La misère engendrée par la guerre civile aurait-elle incité de nombreux parents à se défaire secrètement d'un enfant légitime?
109. Ibid.

110. BARDET Jean-Pierre, "Enfants abandonnés et enfants assistés à Rouen », Annales de démographie historique, 1973, p. 28.

111. Cavallo Sandra,

"Bambini abbandonati e bambini "in deposito" a Torino nel settecento ", Enfance abandonnée et société en Europe..., op. cit., p. 343; Dos Guimaraes Isabel, «The "Casa da Roda do Porto" reception and restitution of foundlings during the eighteenth century ", Enfance abandonnée et société en Europe..., op. cit., p. 544-545; BARDET Jean-Pierre, "La société et l'abandon ", Enfance abandonnée et société en Europe..., op. cit., p. 8; VALVERDE LAMSFus Lola, Entre el deshonor y la miseria, infancia..., op. cit., p. 59-60.

112. De 1798 à 1806, les registres de baptême de la Casa de Misericordia de Bilbao consignent 90 expositions sur 105 abandons (soit 85,7 \%). À l'exception d'un seul dépôt dans le tour de Bilbao, les autres délaissements ont été effectués via la présentation des enfants aux autorités religieuses locales. 
113. AFB, fondo expósitos de Bizkaia, registro 16, 06/02/1816

114. Ces trois communes seront annexées par la capitale provinciale à la fin du XIXe siècle.

115. Les proportions sont: 4,8\% pour Abando, 3,9\% pour Begoña, 2,3 \% pour Deusto et 1,6\% pour Bilbao.

116. Brunet Guy, "Célibataires et mères de nombreux enfants. Parcours de femmes à Lyon au xIxe siècle ", Annales de démographie historique, 2010, n 119, p. 100-102.
Signe que les abandonneurs souhaitent favoriser la découverte des nourrissons, les expositions sont beaucoup plus fréquentes dans les zones urbanisées. Plus excentrées, les fermes sont rarement préférées aux maisons situées dans les centres urbains. Seuls deux bébés sont découverts dans un champ ou sur un chemin. Au final, un seul procès-verbal permet d'établir un lien entre abandon sauvage et infanticide: en 1816, un nouveau-né prénommé Cornelio Serapio est retrouvé à bout de souffle dans un tas d'immondices. Les autorités ne s'y trompent guère et entament des poursuites judiciaires pour faits d'exposition et infanticide ${ }^{113}$.

Une cartographie de l'exposition permet de visualiser la présence d'enfants trouvés sur l'ensemble du territoire provincial (carte 1): sur les 132 localités biscayennes, 94 (soit 71,2 \%) sont confrontées au moins une fois à ce type d'abandon entre 1798 et 1839. Mais Bilbao et les trois agglomérations limitrophes, Deusto, Abando et Begoña ${ }^{114}$, constituent le premier grand foyer d'exposition. Ces quatre villes canalisent plus d'une exposition sur huit $(12,6 \%)^{115}$. Nous pouvons supposer que la volonté d'abandonner son nourrisson à proximité de l'orphelinat provincial est à l'origine de cette surreprésentation. Le phénomène est connu: dans toute l'Europe, les abandons se concentrent autour des maisons d'accueil, de nombreux parents recherchant un lieu disposant d'une institution qui pourra rapidement prendre soin de leur enfant. Mais la ville constitue également une destination privilégiée pour les filles-mères qui souhaitent accoucher en toute discrétion d'un bébé conçu à la campagne. Enfin, la présence en milieu urbain d'une main-d'œuvre féminine qui exerce, loin de tout contrôle familial, une activité professionnelle peu qualifiée et peu rémunérée est propice aux liaisons clandestines, aux relations sexuelles sans lendemain et à leurs conséquences parfois tragiques ${ }^{116}$.

En Biscaye, les expositions sont également particulièrement nombreuses dans la zone minière du Grand Bilbao: les villes de Portugalete, Baracaldo, Musques, Santurce, San Salvador del Valle, Ciérvana et Sestao offrent refuge à $8,5 \%$ des enfants rejetés. À l'inverse, l'abandon est peu répandu dans les zones rurales et maritimes du nord de la province. Dans cet espace géographique, seulement trois villes - les plus importantes - comptabilisent plus de $1 \%$ des délaissements: Guernica (1,6\%), Bermeo (1,2\%) et Munguía (1,2\%). Cette répartition géographique de l'abandon confirme la prédominance des villes dans l'acte d'exposition ainsi que l'origine modeste des parents. 


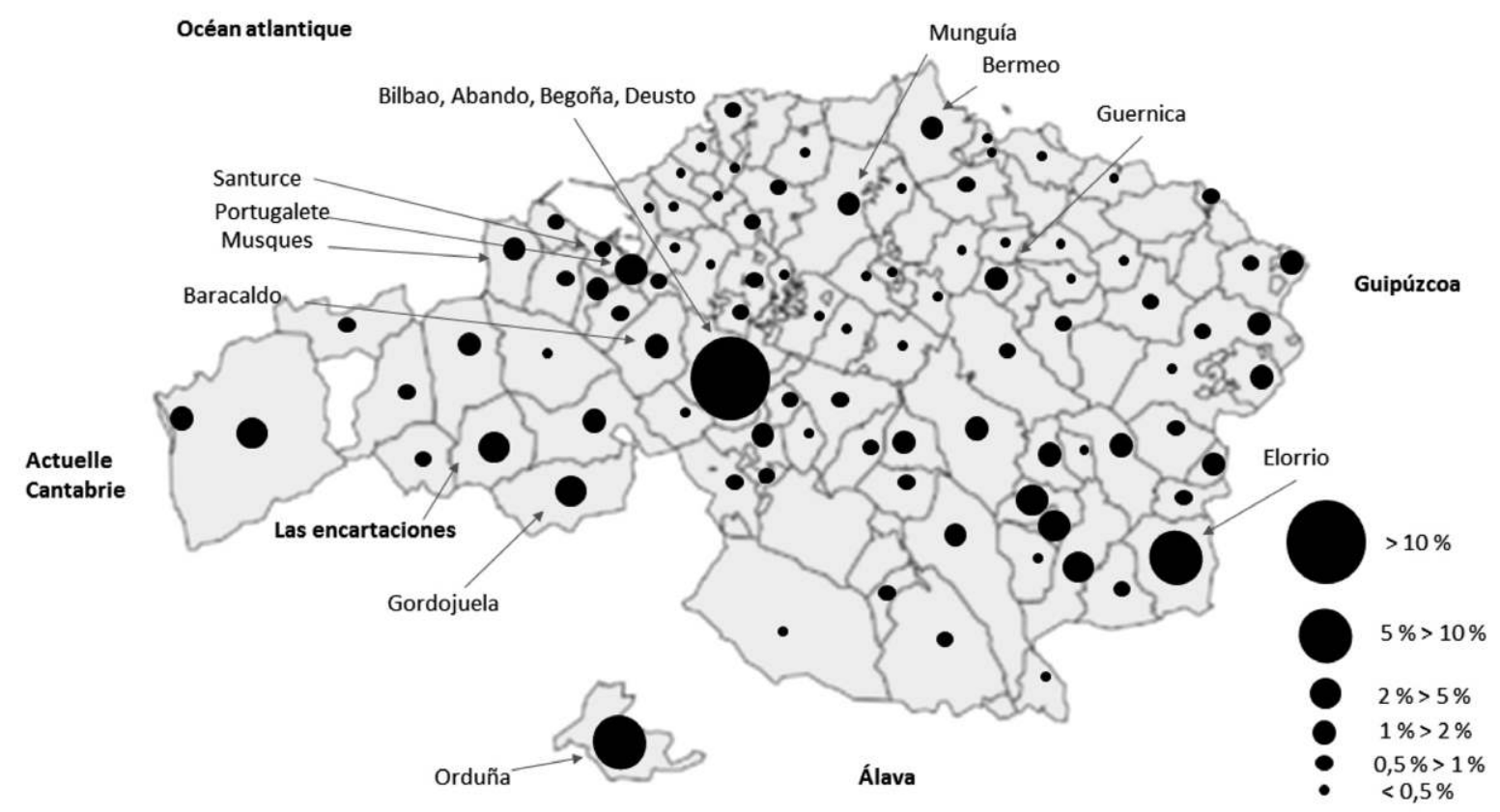

Carte 1: Communes d'exposition des enfants (1798-1839).

Sources: AFB, fondo expósitos de Bizkaia, registros 15, 16, 17, 18, 19, 20, 21.

En marge du Grand Bilbao, deux villes excentrées apparaissent comme des lieux de dépôt privilégiés: Orduña, implantée à la limite de la Castille et de la province d'Alava, concentre à elle-seule 8,8 \% du total des expositions; Elorrio, située à l'extrémité sud-est, à proximité de la province de Guipúzcoa, en recueille $6,7 \%$. Plus généralement, à l'est de la Biscaye, ce sont l'ensemble des villes localisées le long de la frontière du Guipúzcoa qui constituent une importante zone d'exposition: plus d'un enfant sur cinq y est abandonné. De l'autre côté de la Biscaye, Balmesada et la région de Las Encartaciones, qui s'étend à proximité de la Castille, concentrent 12,1\% des délaissements sauvages. Cette prépondérance des zones limitrophes fait écho aux plaintes des responsables de l'enfance délaissée biscayenne qui dénoncent la présence sur le territoire dont ils ont la charge de bébés originaires des provinces de Guipúzcoa, d'Alava et de Castille.

La volonté ou la nécessité de garder l'anonymat pousse donc nombre de parents à délaisser leurs bébés dans les plus grands centres urbains. Signe qưils 
117. AFB, fondo expósitos de Bizkaia, registro 252, reglamento de 1845 . souhaitent fréquemment faciliter leur survie, ils n'hésitent pas à franchir les frontières provinciales pour leur garantir une prise en charge et semblent se rapprocher le plus possible de leur lieu d'accueil. Mais la Maison des Enfants abandonnés de Biscaye parviendra-t-elle à assurer leur survie? Rien n'est moins certain. Pour la période 1807-1814, les autorités biscayennes en charge de l'enfance délaissée annoncent encore un taux de mortalité annuel de 49,9\%: 437 des 875 orphelins accueillis n'ont pas survécu ${ }^{117}$.

Au terme de cette étude, il ne fait aucun doute que, marchant dans le sillage des esprits éclairés français, les autorités espagnoles et biscayennes de la fin du XVIII ${ }^{\mathrm{e}}$ siècle et du début du XIX ${ }^{\mathrm{e}}$ siècle prennent conscience de l'hécatombe provoquée par l'absence de prise en charge des enfants abandonnés. Comme partout en Espagne, la création de structures d'accueil témoigne d'une amélioration des conditions d'accueil et d'entretien de l'enfance délaissée. Mais le sort des orphelins biscayens est encore excessivement précaire. Conséquence du désengagement financier de l'État, la Biscaye ne parvient pas à subvenir aux besoins de nourrissons de plus en plus nombreux et opte pour l'accueil exclusif de l'enfance illégitime. Discriminatoire, cette politique d'assistance oblige les parents légitimes qui ne souhaitent pas ou ne peuvent pas conserver leur enfant à recourir à l'exposition. Paradoxalement, elle génère la surmortalité de nouveau-nés que les autorités provinciales sont censées protéger. Enfin, qu’ils soient naturels ou légitimes, l'abandon des plus petits devient, dans la Biscaye du XIX ${ }^{e}$ siècle, un fait de société indiscutable. 